\title{
Determination of Isoflavones in Soybean Flour by Matrix Solid-Phase Dispersion Extraction and Liquid Chromatography with UV-Diode Array Detection
}

\author{
Antonella Aresta, ${ }^{1}$ Pietro Cotugno, ${ }^{2}$ Federica Massari, ${ }^{1}$ and Carlo Zambonin ${ }^{1}$ \\ ${ }^{1}$ Department of Chemistry, University of Bari “Aldo Moro", Via Orabona 4, 70125 Bari, Italy \\ ${ }^{2}$ CONISMA, Piazzale Flaminio 9, 00196 Roma, Italy \\ Correspondence should be addressed to Carlo Zambonin; carlo.zambonin@uniba.it
}

Received 7 March 2017; Accepted 17 July 2017; Published 13 August 2017

Academic Editor: Vítor Spínola

Copyright (C) 2017 Antonella Aresta et al. This is an open access article distributed under the Creative Commons Attribution License, which permits unrestricted use, distribution, and reproduction in any medium, provided the original work is properly cited.

\begin{abstract}
A new analytical method, based on liquid chromatography (LC) with UV-diode array detection, for the simultaneous determination of daidzein, genistein, and glycitein and their 7-O- $\beta$-D-glucopyranoside (daidzin, genistin, and glycitin, resp.) has been successfully developed. All the calibration curves showed good linearity within the concentration range $0.02-2 \mu \mathrm{g} / \mathrm{ml}$. The limits of detection and quantitation were 0.057 (genistin and glycitein), $0.124 \mu \mathrm{g} / \mathrm{ml}$ (genistein), $0.190 \mu \mathrm{g} / \mathrm{ml}$ (genistin and glycitein), and $0.410 \mu \mathrm{g} / \mathrm{ml}$ (genistein), respectively. Within-day and between-days precision were found not to be significantly different according to an $F$ test; values (\% RSD) ranged from 2.0 to $2.9 \%$. Extraction and clean-up of soybean flour samples were carried out using matrix solid-phase dispersion extraction (MSPD). The main parameters affecting extraction yield, such as dispersant, type and amount of additives, cosorbent, and extractive solvent, were evaluated and optimized. The average recovery values were between 85.7 and 102.6\%. The target isoflavone concentration levels estimated in this work fit existing literature data and were comprised between 39.3 and $345.3 \mu \mathrm{g} / \mathrm{g}$. The whole procedure has proved to be simple, accurate, precise, and cheap.
\end{abstract}

\section{Introduction}

Phytoestrogens are a heterogeneous group of naturally occurring phenolic compounds with estrogen-like activity [1]. It has been proposed that plants control male fertility by using phytoestrogens as part of their natural defense against herbivorous animal overpopulation $[2,3]$.

Isoflavones are bioactive metabolites that constitutes a well-known group of phytoestrogens, the amounts of which increase in the case of plant stress $[3,4]$. In addition, they possess antibiotic and antioxidant properties because of their ability to trap singlet oxygen [5]. Epidemiological studies have suggested a protective effect of isoflavones against a number of adult chronic diseases, including coronary heart disease as well as breast, endometrial, and prostate cancers [6-8]. However, their efficacy has not been fully proven, so surveys on risks and benefits are still unclear $[9,10]$.

Isoflavones are present in many legumes in free forms (aglycones) that conjugate with glucose or carbohydrate moieties $[4,11]$. The unconjugated forms are the bioactive molecules. The ratio between conjugated and unconjugated forms varies substantially in plants. Previous investigations have shown that genistein and its aglycone are the predominant isoflavones in soybean $[10,11]$. Genistein is a specific inhibitor of human DNA topoisomerase II and may possess anticancer activity [12].

Generally, the analytical determinations of phytoestrogens in food have been carried out by LC-UV, LC-MS, or GC-MS [13-20]. Their analysis in complex matrices obviously requires sample pretreatment procedures to simplify the matrix. The most used methods for isoflavone determination are liquid-liquid (LLE) and solid-phase extraction (SPE) [14]. Recently, solid-phase microextraction (SPME) has also been proposed for the determination of some major isoflavones in soy drinks [21]. A simple, low-cost, and relatively quick alternative to the above approaches is matrix solid-phase dispersion (MSPD). It is primarily used because of its flexibility and selectivity, as well as the possibility of performing 
extraction and clean-up in one step, which results in rapid pretreatment and low solvent consumption. MSPD involves disruption of solid and semisolid samples and simultaneous extraction of the analytes using direct mechanical blending of the sample with a solid-phase sorbent, mainly silica gel or alumina [22]. In particular MSPD has been shown to be a good technique for extraction of isoflavonoids in plants used by alternative medicine for health care practices [18-20]. Xiao et al. developed a useful protocol based on MSDP coupled to LC for the determination of daidzein and genistein in soybeans [19], obtaining high extraction efficiencies (greater than $80 \%$ ) regardless of the use of lower amounts of sample and solvents than traditional methods.

In this paper, MSPD has been successfully used for the extraction of the isoflavones daidzein, genistein, and glycitein, as well as their glycosides daidzin, genistin, and glycitin, respectively, from different defatted soybean flours. The main parameters that affect the extraction of the analytes from the matrix, such as sample/sorbent ratio, sorbent/solvent combination used, and volume of elution solvent, have been carefully evaluated and optimized in order to obtain the highest possible recoveries. The instrumental analysis was performed by liquid chromatography with UV-diode array detection.

\section{Materials and Methods}

2.1. Materials. Daidzein ( $4^{\prime}, 7$-dihydroxyisoflavone), genistein $\left(4^{\prime}, 5,7\right.$-trihydroxyisoflavone $)$, glycitein $\left(4^{\prime}, 7\right.$-dihydroxy6-methoxyisoflavone) and their 7-O- $\beta$-D-glucopyranosides (daidzein, genistin, and glycitin, resp.) were from SigmaAldrich (Milano, Italy). Standards purity was $\geq 95 \%$. Stock solutions of each compound $(0.5 \mathrm{mg} / \mathrm{ml})$ were prepared in a methanol/dimethyl sulfoxide (DMSO) mixture $(80: 20, \mathrm{v} / \mathrm{v})$ and stored at $-20^{\circ} \mathrm{C}$ in the dark. More dilute solutions were prepared just before use in the solvent $A$ of the mobile phase, that is, acetonitrile/water $(5: 95, \mathrm{v} / \mathrm{v})$ with $0.2 \%$ formic acid. All solvents (Sigma-Aldrich) were LC grade. The LC mobile phase was filtered through a $0.20 \mu \mathrm{m}$ nylon membrane (LabService Analytica, Bologna, Italy).

2.2. Apparatus. The SPME-LC system consisted of a Spectra System Pump, model P2000 (ThermoQuest, San Jose, CA), equipped with a Rheodyne 7125 injection valve $(20 \mu \mathrm{l}$ injection loop), a SCM1000 on-line solvent degasser (Thermo Separation Products), and a $2.6 \mu \mathrm{m}$ Kinetex C18 $(10 \times 4.6 \mathrm{~mm}$ i.d., Phenomenex, USA) column. The detector was a Spectra System model UV6000LP photodiode array (Thermo Finnigan, San Jose, CA).

2.3. Chromatographic and Detection Conditions. A binary gradient composed of acetonitrile/water $(5: 95, \mathrm{v} / \mathrm{v})$ with $0.2 \%$ formic acid (solvent $\mathrm{A}$ ) and acetonitrile with $0.2 \%$ formic acid (solvent B) was used as mobile phase. The gradient program was: 30 min linear from $90 \%$ A to $40 \% \mathrm{~A}$; $1 \mathrm{~min}$ isocratic at $40 \% \mathrm{~A} ; 1 \mathrm{~min}$ linear to $90 \% \mathrm{~A} ; 5 \mathrm{~min}$ equilibration time. The flow rate was $0.7 \mathrm{ml} / \mathrm{min}$ and the column was kept at room temperature (around $18^{\circ} \mathrm{C}$ ).
Spectra were acquired in the $240-380 \mathrm{~nm}$ range at the apex and on the ascending or descending part of each peak. Peak purity was checked by spectra overlaying after normalization.

2.4. Samples Collection and Pretreatment. Defatted soybean flour samples were purchased from Sigma-Aldrich (SF1) and local supermarkets (made in the European Community and intended for human consumption, SF2-SF6), respectively. Samples were stored at room temperature and kept in a desiccator after opening. Spiked samples (5g each) were prepared in triplicate by adding suitable amounts of standard solutions.

MSPD was performed as follows: $0.05 \mathrm{~g}$ of flour was mixed with $0.5 \mathrm{~g}$ of anhydrous $\mathrm{Na}_{2} \mathrm{SO}_{4}$. Then, $1 \mathrm{~g}$ of glass beads (previously cleaned with ethyl acetate) and $0.2 \mathrm{~g}$ of silica gel sorbent were added to the sample in a mortar and blended for 15 min using a pestle. The mixture was transferred into a syringe barrel equipped with a polypropylene frit, carefully pressed using the syringe plunger, and capped with a second frit. This step was very important to ensure a calibrated flow of the elution solvent. Finally, $2 \mathrm{ml}$ of ethyl acetate with $2 \%$ DMSO was eluted in the syringe and collected in a vial. Then, $0.2 \mathrm{ml}$ of the solution was dried under a gentle stream of nitrogen, reconstituted in solvent A of the mobile phase, and injected into the LC system.

2.5. Statistical Analysis. Data from different experimental groups of soybean flour samples and concentrations of isoflavones estimated were compared by a two-way analyses of variance (ANOVA) and differences at 95\% level of confidence $(p<0.05)$ were considered significant.

\section{Results and Discussion}

The first step of the present work was to investigate factors influencing retention and chromatographic efficiency of the target compounds in order to optimize their separation. LC separation of phytoestrogens has been generally performed [18] using reverse phase columns and mobile phases consisting of methanol (or acetonitrile) and water, containing small amount of acids. In the present work, a core-shell C18 column was adopted and methanol was preferred to acetonitrile as eluent since the latter was the cause of higher column pressures. Furthermore, acidification of the eluent was needed to allow the formation of protonated species; formic acid was chosen as a milder alternative to trifluoroacetic or phosphoric acids. Eventually, the best resolution was achieved using a binary gradient composed of acetonitrile/water with $0.2 \%$ formic acid (solvent $\mathrm{A}$ ) and acetonitrile with $0.2 \%$ formic acid (solvent B). Figure 1 reports an LC-UV/DAD chromatogram relevant to the analysis of the target isoflavones ((1) daidzin, (2) glycitin, (3) genistin, (4) daidzein, (5) glycitein, and (6) genistein) at the concentration level of $2 \mu \mathrm{g} / \mathrm{ml}$ using the optimized mobile phase composition and shows the good resolution achieved for the target compounds.

Calibration curves were then constructed and proved to be linear in the concentration range $0.2-2 \mu \mathrm{g} / \mathrm{ml}$ for all the analytes apart from genistein $(0.4-2.0 \mu \mathrm{g} / \mathrm{ml})$. Correlation 


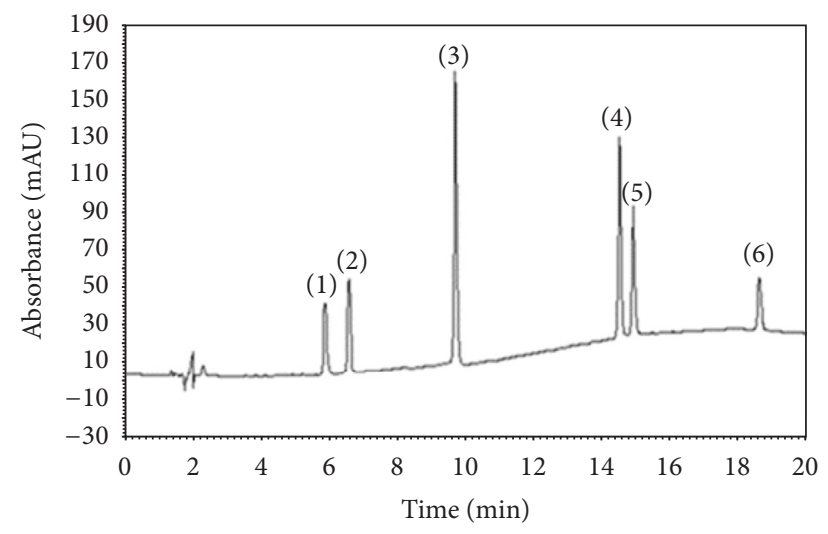

FIGURE 1: LC-UV/DAD chromatogram obtained by the direct injection of a standard solution of the target isoflavones at the concentration level of $2 \mu \mathrm{g} / \mathrm{ml}$. Peak legend: (1) daidzin, (2) glycitin, (3) genistin, (4) daidzein, (5) glycitein, and (6) genistein.

TABLE 1: Estimated LOD and LOQ obtained for the standard isoflavones, together with the precision data of the method.

\begin{tabular}{lccc}
\hline Compound & $\begin{array}{c}\text { LOD } \\
(\mu \mathrm{g} / \mathrm{ml})\end{array}$ & $\begin{array}{c}\text { LOQ } \\
(\mu \mathrm{g} / \mathrm{ml})\end{array}$ & $\begin{array}{c}\text { RSD } \\
(\%)\end{array}$ \\
\hline Daidzin & 0.070 & 0.234 & 2.4 \\
Glycitin & 0.070 & 0.228 & 2.7 \\
Genistin & 0.057 & 0.190 & 2.6 \\
Daidzein & 0.089 & 0.295 & 2.7 \\
Glycitein & 0.057 & 0.190 & 2.0 \\
Genistein & 0.124 & 0.410 & 2.9 \\
\hline
\end{tabular}

coefficients were better than 0.999 and intercepts were not significantly different from zero at the $95 \%$ confidence level. Each concentration was performed in triplicate. The precision of the method was investigated at $0.5 \mu \mathrm{g} / \mathrm{ml}$ by performing replicate measurements $(n=5)$ for 5 days, in order to estimate the within-day and between-days precision, which were found not to be significantly different according to an $F$-test and always lower than $3 \%$. The relevant results are reported in Table 1 . The estimated limits of detection (LODs) and limits of quantitation (LOQs), calculated as 3- and 10fold the standard deviations of the intercept of the calibration curves, are also listed in Table 1.

Experiments were then focused on the optimization of the MSPD procedure. The selectivity of MSPD depends on many variables, that is, sample/sorbent ratio, sorbent/solvent combination used, and volume of elution solvent. In the present case, a ratio of $0.1: 1: 2: 0.4$ between sample, $\mathrm{Na}_{2} \mathrm{SO}_{4}$, glass beads, and sorbent (silica gel), respectively, was optimized to obtain a homogeneous mixture and satisfactory extraction yields. The choice of the nature and the amount of the elution solvent was also crucial since the target analytes must be desorbed while the higher number of the remaining matrix components should be retained in the column. Different types and volumes of solvents were tested and $2 \mathrm{ml}$ of ethyl acetate proved to be the most efficient combination. An increase in analyte solubility in the elution

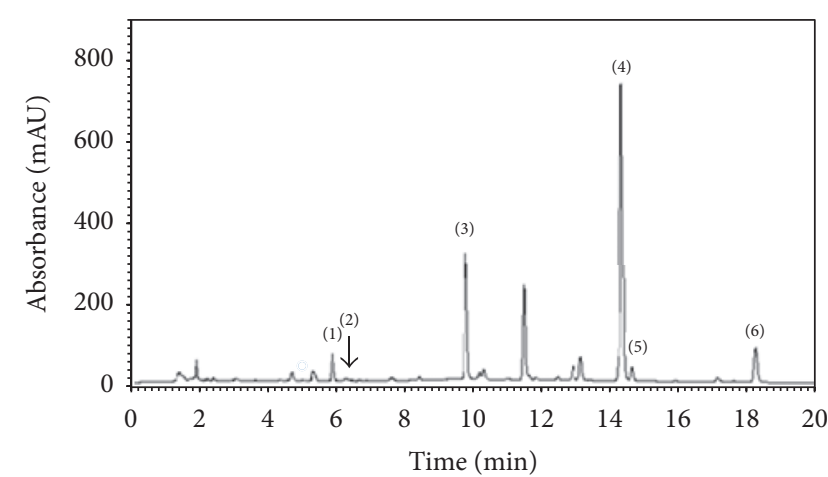

FIGURE 2: LC-UV/DAD chromatogram relevant to the analysis of a soybean flour sample extract. Peak legend: (1) daidzin, (2) glycitin, (3) genistin, (4) daidzein, (5) glycitein, and (6) genistein.

solvent was also achieved by adding $2 \%$ of DMSO, thus obtaining even higher recoveries.

The method was then applied to the determination of the target analytes in different soybean flour samples. A typical chromatogram relevant to the analysis of a soybean flour sample extract is shown in Figure 2. As can be seen, the peaks of the detected analytes were yet again well resolved, with no significant interference from matrix compounds, with the only exception of glycitin, which was never detected in any of the analyzed samples according to literature data [11, 12]. Each chromatographic peak was confirmed by its retention time and the spectra overlaying technique.

Recoveries were calculated by using two spiked flours (FS1 and FS2) at different concentration levels, and the relevant data are reported in Table 2. High recoveries were obtained for all the target isoflavones, thus confirming the good extraction efficiency of the developed MSPD procedure. The within-day $(n=3)$ coefficients of variation, also reported in Table 2, calculated in the same samples (FS1 and FS2) at the same concentration levels were always below $6 \%$.

Quantitation was performed with the standard addition method. Table 3 lists the isoflavone concentration levels estimated in the selected soybean flour samples. Typically, the target concentration levels of isoflavones in soybeans and derived products range between 0.1 and $5 \mathrm{mg} / \mathrm{g}$ [15]. Accordingly, the levels estimated in this work were between 39.3 and $345.3 \mu \mathrm{g} / \mathrm{g}$. It is worth noting that the variation of the concentration levels observed between the different soybean flours is not statistically significant, as confirmed by a two-way ANOVA test. Soybean flours can be made in different ways: in the traditional East Asian way by dryroasting soybeans or in the modern Western way involving moist heating. Andrade et al. proved that the choice of oven can modify the content and profile of the different isoflavone forms in soybean flour [23]. Since the analysed samples were obtained using the same process, the results are not surprising. Furthermore, a recent paper reports that significant variances in chemical composition, protein profile, and isoflavone contents can be found between soybean cultivars, determining important differences in their nutritional and functional values [24]. Our experimental results obtained in 
TABLE 2: Percentage recoveries of isoflavones obtained from two spiked soybean flours $(n=3)$ at different concentration levels, together with the within-day $(n=3)$ coefficients of variation.

\begin{tabular}{|c|c|c|c|c|c|c|c|}
\hline Sample & Isoflavone & $\begin{array}{c}\text { Spiked concentration } \\
(\mu \mathrm{g} / \mathrm{g})\end{array}$ & $\begin{array}{c}\text { \% recovery mean } \\
\pm \mathrm{SD}\end{array}$ & $\begin{array}{l}\text { Precision } \\
(\% \mathrm{RSD})\end{array}$ & $\begin{array}{c}\text { Spiked concentration } \\
(\mu \mathrm{g} / \mathrm{g})\end{array}$ & $\begin{array}{l}\% \text { recovery } \\
\text { mean } \pm \mathrm{SD}\end{array}$ & $\begin{array}{c}\text { Precision } \\
(\% \text { RSD) }\end{array}$ \\
\hline \multirow{6}{*}{ FS1 } & Daidzin & 70 & $88.2 \pm 4.9$ & 5.6 & 700 & $87.8 \pm 4.3$ & 4.9 \\
\hline & Glycitin & 40 & $88.4 \pm 10.3$ & 5.7 & 400 & $88.6 \pm 3.9$ & 4.4 \\
\hline & Genistin & 100 & $102.6 \pm 6.1$ & 5.9 & 1000 & $98.5 \pm 4.9$ & 5.0 \\
\hline & Daidzein & 100 & $85.7 \pm 4.0$ & 4.7 & 1000 & $86.2 \pm 5.1$ & 5.9 \\
\hline & Glycitein & 50 & $87.5 \pm 3.9$ & 4.5 & 500 & $86.7 \pm 4.2$ & 4.8 \\
\hline & Genistein & 330 & $95.0 \pm 4.2$ & 4.4 & 3300 & $93.8 \pm 3.9$ & 4.2 \\
\hline \multirow{6}{*}{$\mathrm{FS} 2$} & Daidzin & 70 & $88.1 \pm 5.0$ & 5.7 & 700 & $86.4 \pm 4.9$ & 5.7 \\
\hline & Glycitin & 40 & $86.4 \pm 9.5$ & 5.9 & 400 & $88.6 \pm 5.2$ & 5.9 \\
\hline & Genistin & 100 & $99.3 \pm 4.9$ & 4.9 & 1000 & $88.5 \pm 5.1$ & 5.8 \\
\hline & Daidzein & 100 & $86.0 \pm 3.4$ & 4.0 & 1000 & $87.2 \pm 4.9$ & 5.6 \\
\hline & Glycitein & 50 & $100.5 \pm 5.3$ & 5.3 & 500 & $98.4 \pm 4.5$ & 4.6 \\
\hline & Genistein & 330 & $90.0 \pm 5.0$ & 5.6 & 3300 & $99.8 \pm 5.6$ & 5.6 \\
\hline
\end{tabular}

TABLE 3: Concentrations of isoflavones estimated $(n=3)$ in the selected soybean flour samples.

\begin{tabular}{|c|c|c|c|c|c|c|c|}
\hline Sample & $\begin{array}{c}\text { Daidzin } \\
(\mu \mathrm{g} / \mathrm{g})\end{array}$ & $\begin{array}{c}\text { Glycitin } \\
(\mu \mathrm{g} / \mathrm{g})\end{array}$ & $\begin{array}{c}\text { Genistin } \\
(\mu \mathrm{g} / \mathrm{g})\end{array}$ & $\begin{array}{c}\text { Daidzein } \\
(\mu \mathrm{g} / \mathrm{g})\end{array}$ & $\begin{array}{c}\text { Glycitein } \\
(\mu \mathrm{g} / \mathrm{g})\end{array}$ & $\begin{array}{c}\text { Genistein } \\
(\mu \mathrm{g} / \mathrm{g})\end{array}$ & $\begin{array}{l}\text { Total } \\
(\mu \mathrm{g} / \mathrm{g}) \\
\end{array}$ \\
\hline FS1 & $68.0 \pm 1.6$ & nd & $102.4 \pm 2.1$ & $96.0 \pm 1.7$ & $44.4 \pm 1.1$ & $320.0 \pm 7.2$ & 631.2 \\
\hline FS2 & $69.0 \pm 1.3$ & nd & $100.0 \pm 1.9$ & $100.0 \pm 1.9$ & $50.0 \pm 1.2$ & $330.0 \pm 7.4$ & 649.5 \\
\hline FS3 & $66.7 \pm 1.5$ & nd & $92.0 \pm 2.0$ & $98.0 \pm 1.9$ & $44.8 \pm 1.0$ & $345.3 \pm 6.6$ & 647.1 \\
\hline FS4 & $70.0 \pm 1.5$ & nd & $99.0 \pm 1.5$ & $96.1 \pm 1.7$ & $42.9 \pm 1.0$ & $290.7 \pm 7.5$ & 599.0 \\
\hline FS5 & $80.2 \pm 1.7$ & nd & $110.4 \pm 2.0$ & $89.7 \pm 1.8$ & $50.0 \pm 1.5$ & $310.0 \pm 6.9$ & 640.6 \\
\hline FS6 & $74.5 \pm 1.6$ & nd & $112.0 \pm 2.2$ & $95.4 \pm 1.7$ & $39.3 \pm 1.1$ & $318.9 \pm 8.4$ & 640.5 \\
\hline
\end{tabular}

selected samples, all purchased in Italy, indicate that they could arise from the same cultivar.

\section{Conclusions}

In the agro-food sector, fast, sensitive, and reliable analytical methodologies are required in order to allow the matrix components in complex samples, such as soybean flours, not only to be highlighted but also to be quantified. These methods should also meet logistic requirements, that is, being accurate, precise, easily transferable, and usable also in low-technology laboratories for use in the area of manufacturing, importing, or acceptance of raw materials. A selective MSPD-LC-UV/DAD analytical method has been successfully developed and validated for the analysis of the isoflavones daidzein, genistein, and glycitein, as well as their glucosides in soybean flour. The results presented in the paper show that MSPD is a very simple, accurate, precise, and cheap sample preparation procedure, suitable for the isolation of isoflavones from soybean products. The target compounds were determined in six different soybean flours, obtained using the same process, and very similar results were obtained, likely indicating a common origin for the samples. According to literature data, genistein and its glucoside are the most abundant forms found in analyzed samples.

\section{Conflicts of Interest}

The authors declare there are no conflicts of interest regarding the publication of this paper.

\section{References}

[1] C. S. Hwang, H. S. Kwak, H. J. Lim et al., "Isoflavone metabolites and their in vitro dual functions: They can act as an estrogenic agonist or antagonist depending on the estrogen concentration," Journal of Steroid Biochemistry and Molecular Biology, vol. 101, no. 4-5, pp. 246-253, 2006.

[2] C. L. Hughes Jr., "Phytochemical mimicry of reproductive hormones and modulation of herbivore fertility by phytoestrogens," Environmental Health Perspectives, vol. 78, pp. 171-175, 1988.

[3] W. Barz and R. Welle, "Biosynthesis and metabolism of isoflavones and pterocarpan phytoalexins in chickpea, soybean and phytopathogenic fungi," Phenolic Metabolism in Plants Recent Advances in Phytochemistry, vol. 26, pp. 139-164, 1992.

[4] R. A. Dixon, "Natural products and plant disease resistance," Nature, vol. 411, no. 6839, pp. 843-847, 2001.

[5] K. B. Pandey and S. I. Rizvi, "Plant polyphenols as dietary antioxidants in human health and disease," Oxidative Medicine and Cellular Longevity, vol. 2, no. 5, pp. 270-278, 2009.

[6] C. D. Haines, P. A. Harvey, E. D. Luczak et al., "Estrogenic compounds are not always cardioprotective and can be lethal in 
males with genetic heart disease," Endocrinology, vol. 153, no. 9, pp. 4470-4479, 2012.

[7] E. Zhao and Q. Mu, "Phytoestrogen biological actions on mammalian reproductive system and cancer growth," Scientia Pharmaceutica, vol. 79, no. 1, pp. 1-20, 2011.

[8] L. Vitetta, A. Sali, and S. Coulson, "Soy and health: The latest evidence," Medicine Today, vol. 12, no. 3, pp. 67-74, 2011.

[9] S. Andres, K. Abraham, K. E. Appel, and A. Lampen, "Risks and benefits of dietary isoflavones for cancer," Critical Reviews in Toxicology, vol. 41, no. 6, pp. 463-506, 2011.

[10] M. Messina and V. L. Messina, "Exploring the soyfood controversy," Nutrition Today, vol. 48, no. 2, pp. 68-75, 2013.

[11] S. Barnes, "The biochemistry, chemistry and physiology of the isoflavones in soybeans and their food products," Lymphatic Research and Biology, vol. 8, no. 1, pp. 89-98, 2010.

[12] Y. Mizushina, K. Shiomi, I. Kuriyama, Y. Takahashi, and H. Yoshida, "Inhibitory effects of a major soy isoflavone, genistein, on human DNA topoisomerase II activity and cancer cell proliferation," International Journal of Oncology, vol. 43, no. 4, pp. 1117-1124, 2013.

[13] Q. Wu, M. Wang, and J. E. Simon, "Analytical methods to determine phytoestrogenic compounds," Journal of Chromatography B: Analytical Technologies in the Biomedical and Life Sciences, vol. 812, no. 1-2, pp. 325-355, 2004.

[14] M. A. Rostagno, A. Villares, E. Guillamón, A. García-Lafuente, and J. A. Martínez, "Sample preparation for the analysis of isoflavones from soybeans and soy foods," Journal of Chromatography A, vol. 1216, no. 1, pp. 2-29, 2009.

[15] S. Barnes, M. Kirk, and L. Coward, "Isoflavones and their conjugates in soy foods: Extraction conditions and analysis by HPLC-mass spectrometry," Journal of Agricultural and Food Chemistry, vol. 42, no. 11, pp. 2466-2474, 1994.

[16] A. Zafra-Gómez, A. Garballo, L. E. García-Ayuso, and J. C. Morales, "Improved sample treatment and chromatographic method for the determination of isoflavones in supplemented foods," Food Chemistry, vol. 123, no. 3, pp. 872-877, 2010.

[17] G. Fiechter, B. Raba, A. Jungmayr, and H. K. Mayer, "Characterization of isoflavone composition in soy-based nutritional supplements via ultra performance liquid chromatography," Analytica Chimica Acta, vol. 672, no. 1-2, pp. 72-78, 2010.

[18] B. Barfi, M. R. Hadjmohammadi, and M. R. Kasaai, "Determination of daidzein and genistein in soybean and its waste by matrix solid-phase dispersion extraction and HPLC," Monatshefte fur Chemie, vol. 140, no. 10, pp. 1143-1148, 2009.

[19] H. B. Xiao, M. Krucker, K. Albert, and X. M. Liang, "Determination and identification of isoflavonoids in Radix astragali by matrix solid-phase dispersion extraction and highperformance liquid chromatography with photodiode array and mass spectrometric detection," Journal of Chromatography A, vol. 1032, no. 1-2, pp. 117-124, 2004.

[20] E. de Rijke, F. de Kanter, F. Ariese, U. A. T. Brinkman, and C. Gooijer, "Liquid chromatography coupled to nuclear magnetic resonance spectroscopy for the identification of isoflavone glucoside malonates in T. pratense L. leaves," Journal of Separation Science, vol. 27, no. 13, pp. 1061-1070, 2004.

[21] A. Aresta, F. Di Grumo, and C. Zambonin, "Determination of Major Isoflavones in Soy Drinks by Solid-Phase Micro Extraction Coupled to Liquid Chromatography," Food Analytical Methods, vol. 9, no. 4, pp. 925-933, 2016.

[22] S. A. Barker, "Matrix solid phase dispersion (MSPD)," Journal of Biochemical and Biophysical Methods, vol. 70, no. 2, pp. 151-162, 2007.
[23] J. C. Andrade, J. M. G. Mandarino, L. E. Kurozawa, and E. I. Ida, "The effect of thermal treatment of whole soybean flour on the conversion of isoflavones and inactivation of trypsin inhibitors," Food Chemistry, vol. 194, pp. 1095-1101, 2016.

[24] S. Ciabotti, A. C. B. B. Silva, A. C. P. Juhasz et al., "Chemical composition, protein profile, and isoflavones content in soybean genotypes with different seed coat colors," International Food Research Journal, vol. 23, no. 2, pp. 621-629, 2016. 

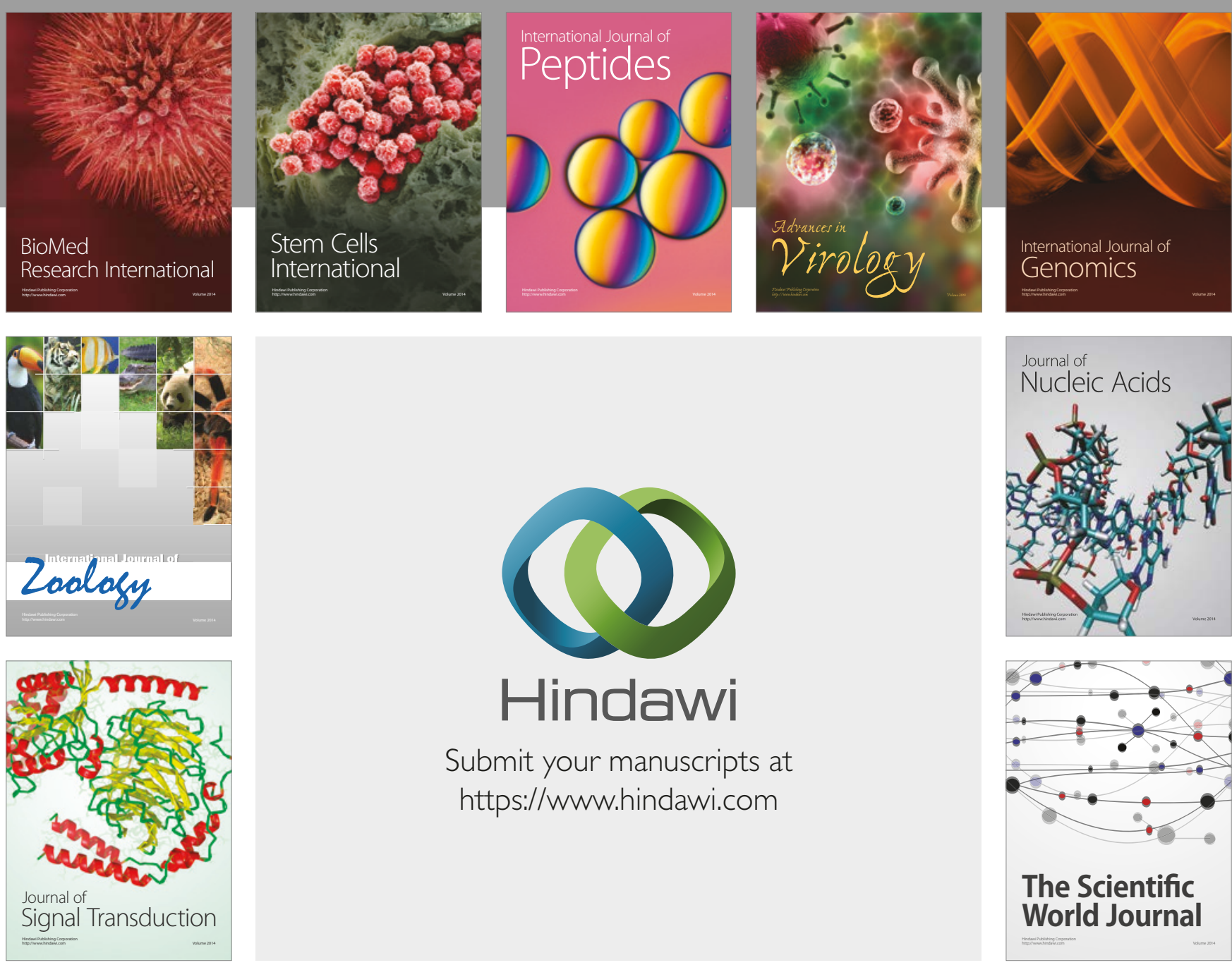

Submit your manuscripts at

https://www.hindawi.com
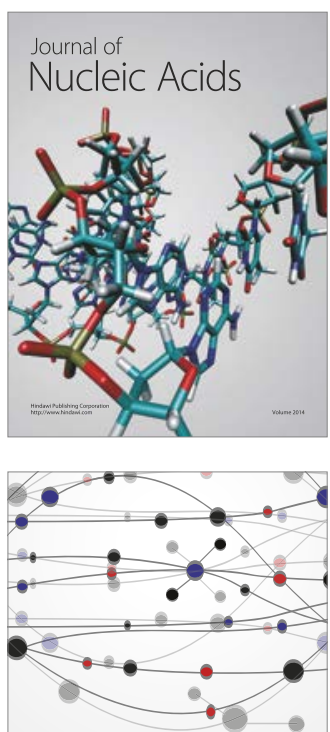

The Scientific World Journal

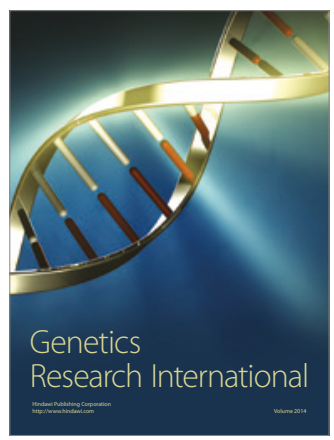

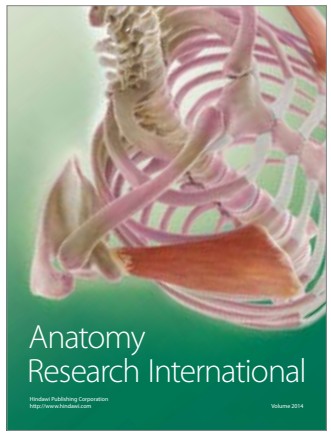

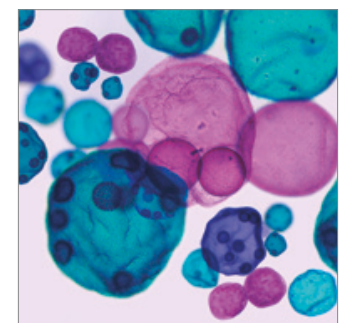

International Journal of Microbiology
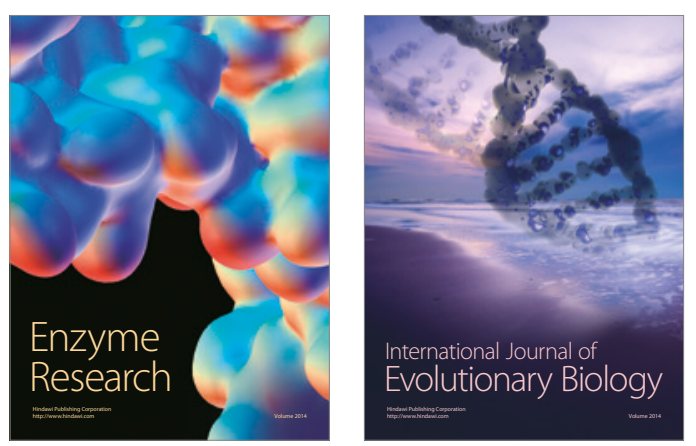
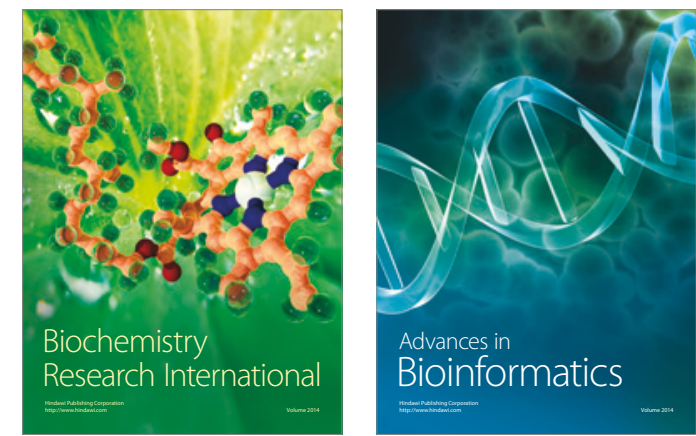

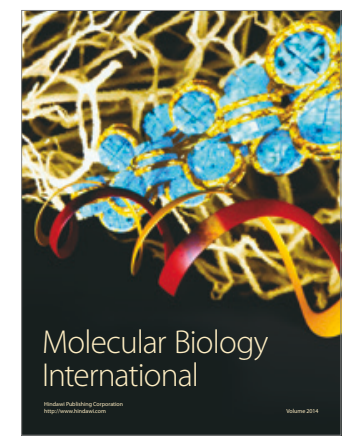

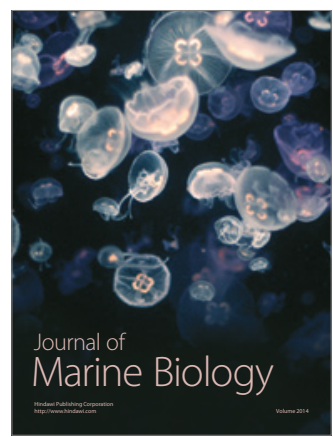

